\title{
Sequestration of Polo kinase to microtubules by phosphopriming-independent binding to Map205 is relieved by phosphorylation at a CDK site in mitosis
}

\author{
Vincent Archambault, ${ }^{1,3}$ Pier Paolo D'Avino, ${ }^{1}$ Michael J. Deery, ${ }^{2}$ Kathryn S. Lilley, ${ }^{2}$ \\ and David M. Glover ${ }^{1,4}$ \\ ${ }^{1}$ Department of Genetics, University of Cambridge, Cambridge, CB2 3EH, United Kingdom; ${ }^{2}$ Department of Biochemistry, \\ University of Cambridge, Cambridge, CB2 1GA, United Kingdom
}

\begin{abstract}
The conserved Polo kinase controls multiple events in mitosis and cytokinesis. Although Polo-like kinases are regulated by phosphorylation and proteolysis, control of subcellular localization plays a major role in coordinating their mitotic functions. This is achieved largely by the Polo-Box Domain, which binds prephosphorylated targets. However, it remains unclear whether and how Polo might interact with partner proteins when priming mitotic kinases are inactive. Here we show that Polo associates with microtubules in interphase and cytokinesis, through a strong interaction with the microtubule-associated protein Map205. Surprisingly, this interaction does not require priming phosphorylation of Map205, and the Polo-Box Domain of Polo is required but not sufficient for this interaction. Moreover, phosphorylation of Map205 at a CDK site relieves this interaction. Map205 can stabilize Polo and inhibit its cellular activity in vivo. In syncytial embryos, the centrosome defects observed in polo hypomorphs are enhanced by overexpression of Map205 and suppressed by its deletion. We propose that Map205-dependent targeting of Polo to microtubules provides a stable reservoir of Polo that can be rapidly mobilized by the activity of Cdk1 at mitotic entry.
\end{abstract}

[Keywords: Polo; cell cycle; Drosophila; kinase; Map205; mitosis]

Supplemental material is available at http://www.genesdev.org.

Received May 9, 2008; revised version accepted August 7, 2008.

Control of the eukaryotic cell division cycle requires the regulated activity of several kinases, including cyclindependent kinases (Cdks) and Polo-like kinases (Plks). Drosophila Polo (Sunkel and Glover 1988; Llamazares et al. 1991) is the founding member of the Plk family. Of the four human Plks (Plk1-4), Plk1 is the closest ortholog of Polo in both sequence and function and the best characterized of the vertebrate Plks (Barr et al. 2004; van de Weerdt and Medema 2006). Polo and its orthologs have been implicated in an extensive list of functions in cell division (Barr et al. 2004; Glover 2005; van de Weerdt and Medema 2006) that include enabling mitotic entry by phosphorylating the Cdc25C phosphatase (Kumagai and Dunphy 1996); facilitating loss of sister chromatid cohesion (Alexandru et al. 2001; Sumara et al. 2002; Clarke et

Corresponding authors.

${ }^{3}$ E-MAIL va228@cam.ac.uk; FAX 44-0-1223-333992.

${ }^{4}$ E-MAIL dmg25@hermes.cam.ac.uk; FAX 44-0-1223-333992.

Article is online at http://www.genesdev.org/cgi/doi/10.1101/gad.486808. al. 2005); centrosome separation and maturation (Sunkel and Glover 1988; Lane and Nigg 1996; Casenghi et al. 2003; Oshimori et al. 2006); establishment of the bipolar spindle (Sunkel and Glover 1988; Sumara et al. 2004; van Vugt et al. 2004); tension sensing at the kinetochore in the spindle attachment checkpoint (Ahonen et al. 2005; Wong and Fang 2005, 2007); and cytokinesis (Ohkura et al. 1995; Carmena et al. 1998; Seong et al. 2002; Neef et al. 2003, 2007; Burkard et al. 2007; Petronczki et al. 2007).

This multiplicity of functions requires Polo kinases to be precisely regulated in space and time. Indeed, the cellular localization of Polo kinases changes drastically as the cell cycle progresses (Llamazares et al. 1991; Golsteyn et al. 1995; Moutinho-Santos et al. 1999). To this end, Polo kinases possess a C-terminal Polo-Box Domain (PBD) (Elia et al. 2003a,b; Lowery et al. 2004) that functions in targeting Polo kinases to centrosomes, mitotic kinetochores, and the cytokinetic midbody (Seong et al. 2002; Park et al. 2004). The PBD alone is able to interact 
with a plethora of cellular phosphoproteins (Lowery et al. 2007), and many interactions require the binding of the PBD to a phospho-serine or -theronine of the motif S-pS or S-pT (Elia et al. 2003b; van de Weerdt and Medema 2006). Cyclin B-Cdk1 kinase activity is often responsible for priming these Polo docking sites. Phosphorylation of INCENP (Goto et al. 2006) and BubR1 (Wong and Fang 2007) by Cdk1 promotes the binding of hPlk1 to those targets at the kinetochore. In other cases, Polo kinase primes its own binding. Thus, phosphorylation of PIBP1 by hPlk1 promotes a stable interaction between the two proteins, also at the kinetochore (Kang et al. 2006). hPlk1 also primes its own binding to the motor protein MKlp2 on the central spindle in anaphase/telophase (Neef et al. 2003). Recently, it has been shown that Cdk1 phosphorylation of the central spindle protein PRC1 in early mitosis prevents its binding to hPlk1, while hPlk1 primes its own binding to PRC1 in anaphase. It was proposed that inactivation of cyclin B-Cdk1 at the metaphase-anaphase transition controls the translocation of hPlk1 from centrosomes and kinetochores to the central spindle and midbody, where it is required for cytokinesis (Neef et al. 2007). Polo is also targeted to the central spindle by Feo, the Drosophila counterpart of PRC1 (D'Avino et al. 2007). However, the PRC1 residues targeted by Plk1 are not conserved in Feo, suggesting an alternative mechanism for these proteins to interact. Localization of hPlk1 to centrosomes was also reported to depend on a PBD capable of binding phosphorylated targets, and that hPlk1 binding to Cdc25C depends on its phosphorylation (Elia et al. $2003 \mathrm{~b}$. However, recent results suggest that hPlk1 does not require a functional PBD to localize to centrosomes and that the PBD can interact with a Cdc25C peptide in vitro regardless of its phosphorylation state (Garcia-Alvarez et al. 2007). Together these data indicate that although priming phosphorylation strongly promotes docking of Plks to their targets, it may not be necessary for all interactions.

Polo kinases are also regulated by phosphorylation and proteolysis, and their activity peaks in mitosis (Fenton and Glover 1993; Golsteyn et al. 1995). Human Plk1 and Xenopus Plx1 have both been shown to be activated in mitosis by phosphorylation at a threonine residue (T210 in hPlk1) that is conserved in Drosophila Polo and in other Plks (Qian et al. 1999; Kelm et al. 2002). In human cells, this activating phosphorylation is mediated by Aurora A and its adaptor protein Bora (Macurek et al. 2008; Seki et al. 2008). hPlk1 levels are cell cycle-regulated by an APC-dependent ubiquitin-proteasome pathway (Ferris et al. 1998; Lindon and Pines 2004), peaking in early mitosis and reaching its lowest levels in G1 (Lindon and Pines 2004). This degradation requires a destruction box that is conserved in Drosophila Polo (Lindon and Pines 2004). However, degradation of Plk1 is relatively inefficient, and substantial levels of Plk1 remain in interphase (Lindon and Pines 2004). What happens to the interphase pool of Polo or Plk1 is unknown.

Here we show that Drosophila Polo is targeted to microtubules (MTs) in cytokinesis and interphase. This is mediated through a strong interaction between Polo and Map205, a MT-associated protein. Although this interaction depends on a functional PBD, it does not require priming phosphorylation of Map205, demonstrating the ability of Plks to engage in phosphorylation-independent interactions. We show that phosphorylation of Map205 at a Cdk1 site releases Polo from MTs and that Map205 can stabilize Polo and inhibit its cellular activity. We propose that Cdk1 phosphorylation of Map205 at mitotic entry releases Polo from its inhibitory sequestration to MTs to promote its cellular activities in mitosis. This mechanism provides a novel layer of spatio-temporal control of Polo in the cell cycle.

\section{Results}

Polo localizes to MTs in interphase and cytokinesis

To study Polo's dynamic localization, we generated a cell line stably expressing Polo-GFP (D'Avino et al. 2007). Consistent with previous studies in the whole organism, this cell line revealed the dynamic association of Polo with mitotic kinetochores and centrosomes and with the cleavage furrow and midbody in cytokinesis (Fig. 1A,B; Logarinho and Sunkel 1998). In addition, we found that Polo strongly localized to interphase and central spindle MTs (Fig. 1A-C). The interphase colocalization of Polo-GFP with $\alpha$-tubulin was particularly clear in cells showing a cortical array of MTs (Fig. 1B) (typical of a large proportion of D-Mel cells). This localization of Polo-GFP to MTs in interphase cells was confirmed by plating cells on a concanavalin A-coated surface, allowing them to spread and promote the formation of long MTs (Fig. 1C; Rogers et al. 2002). Time-lapse analysis (Supplemental Movie S1) showed that Polo-GFP localized on MTs emanating from centrosomes in prophase (Fig. 1A, $\mathrm{T}_{0}$, top left cell) and became almost invisible on MTs from prometaphase to anaphase (Fig. 1A, 8:40-36:40, top left cell). Polo-GFP progressively reappeared on MTs in telophase (Fig. 1A, 45:40-50:00, top left cell) and became strongly localized to the central spindle in cytokinesis (Fig. 1A, $\mathrm{T}_{0}-8: 40$, bottom right cell). Fixed cells displayed a very similar Polo distribution pattern (Fig. 1B; Supplemental Fig. S1). To verify if Polo also localizes to MTs in the whole animal, we examined embryos from a transgenic Drosophila line expressing GFP-Polo (Moutinho-Santos et al. 1999). Like in cultured cells, GFP-Polo localized to centrosomes, kinetochores, and the central spindle, but also strongly accumulated to the nuclear envelope in this tissue (Fig. 1D; Moutinho-Santos et al. 1999). Because the nuclear envelope fenestrates but remains around the spindle during early mitosis in Drosophila embryos and since GFP-Polo is enriched at the nuclear envelope, it is difficult to assess its localization to MTs in early mitosis. However, GFP-Polo was clearly visible on MTs of the central spindle during karyokinesis in the syncytial embryo (Fig 1D, arrows). Therefore, Polo kinase is recruited onto MTs in interphase and cytokinesis in cultured cells and during karyokinesis in embryos. 
A

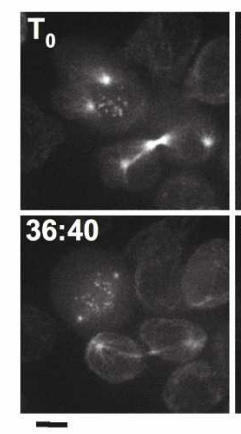

B

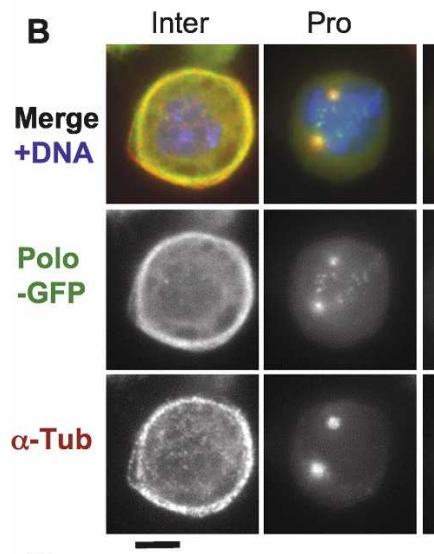

D
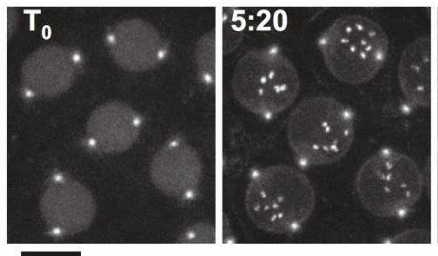

\section{C}
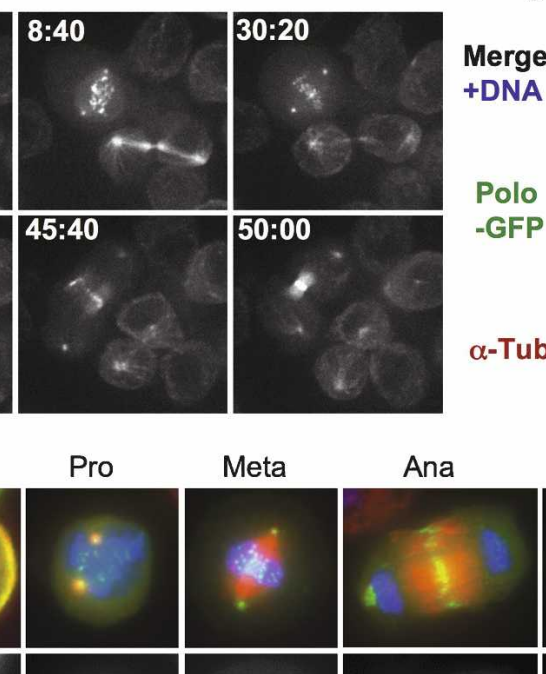

Ana
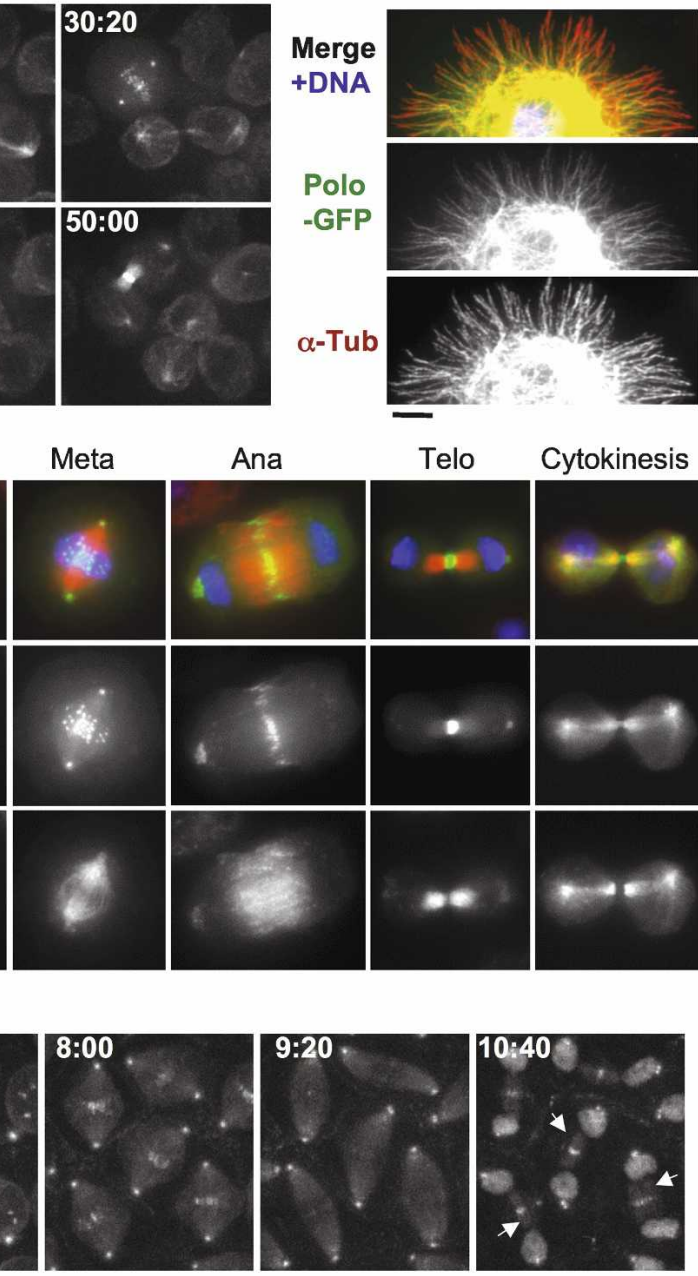

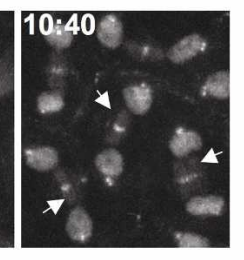

Figure 1. Polo localizes to MTs in a cell cycle-dependent manner. In addition to centrosomes, kinetochores, and the midbody, Polo-GFP localizes to MTs in cytokinesis and interphase. (A) Polo-GFP dynamics in DMel cells by time lapse (time is shown in minutes:seconds). Bar, $5 \mu \mathrm{m}$. See Supplemental Movie 1 and the text for details. (B) PoloGFP colocalizes with MTs during interphase and cytokinesis. Cells were fixed with formaldehyde. Polo-GFP appears in green, $\alpha-\mathrm{Tu}-$ bulin is stained in red, and DNA is DAPIstained in blue. Bar, $5 \mu \mathrm{m}$. (C) Polo-GFP colocalizes with MTs in a cell spreading on a concanavalin-A-coated surface and fixed with methanol/formaldehyde (only a portion of the cell is shown for clarity). Stainings are anti-GFP (green), $\alpha$-Tubulin (red), and DNA (blue). Bar, $5 \mu \mathrm{m}$. (D) GFP-Polo dynamics in a transgenic syncytial embryo (cycle 13) by time lapse (time is shown in minutes:seconds). Note that GFP-Polo localizes to the MTs of the central spindle during karyokinesis (arrows). Bar, $10 \mu \mathrm{m}$.
Polo's targeting to MTs requires an interaction with Map205

To identify binding partners of Polo, we stably expressed Polo in fusion with two IgG-binding domains of the Protein A from Staphylococcus aureus (Polo-PrA) and carried out purifications by affinity of the PrA tag for rabbit IgG. We then identified the associated proteins by mass spectrometry. The strongest band was identified as the MT-associated protein Map205 (Fig. 2A). This interaction was specific since Map205 was not isolated in purifications of unrelated proteins in parallel and under the same conditions. Furthermore, the apparent stoichiometry and the absence of other bands of similar intensity strongly suggested a direct interaction with Polo (Fig. 2A). Reciprocally, endogenous Polo specifically copurified with PrA-Map205 (Fig. 2B). Direct immunoprecipitation of endogenous Map205 confirmed its interaction with endogenous Polo, in both D-Mel cells and embryos (Fig. 2C). Cofractionation of Polo with Map205 was also recently shown in a sucrose gradient analysis of Drosophila MT-binding proteins prepared from embryos (Sorokina and Kashina 2005). Map205 is a functionally conserved MT-associated protein /Goldstein et al. 1986;
Maiato et al. 2004). Its orthologs in human (Map4), Xenopus (XMap230), and budding yeast (Mhp1) are all required for normal MT dynamics in interphase and mitosis (Goldstein et al. 1986; Cha et al. 1999; Andersen 2000; Maiato et al. 2004). Since Map205 localizes to MTs throughout the cell cycle (Goldstein et al. 1986) and Polo localizes to MTs in interphase and cytokinesis (Fig. $1)$, it is not surprising that we found that the two colocalized in interphase and cytokinesis (Supplemental Fig. S2).

We hypothesized that Map205 could be required for the recruitment of Polo to MTs. To test this, we depleted Map205 by RNAi in cultured cells (Fig. 2D,E). Interestingly, Polo levels were reduced following depletion of Map205, suggesting that the interaction with Map205 may stabilize Polo. Consistent with this, we found that RNAi depletion of Map205 altered the cell cycle profile as revealed by FACS and led to an increase in mitotic index accompanied by an accumulation of cells in prometaphase (Supplemental Fig. S3). We verified that Polo destabilization following Map205 depletion was caused by the loss of interaction between Polo and Map205 and not from other perturbations arising from the loss of Map205, by replacing the endogenous Map205 with a 


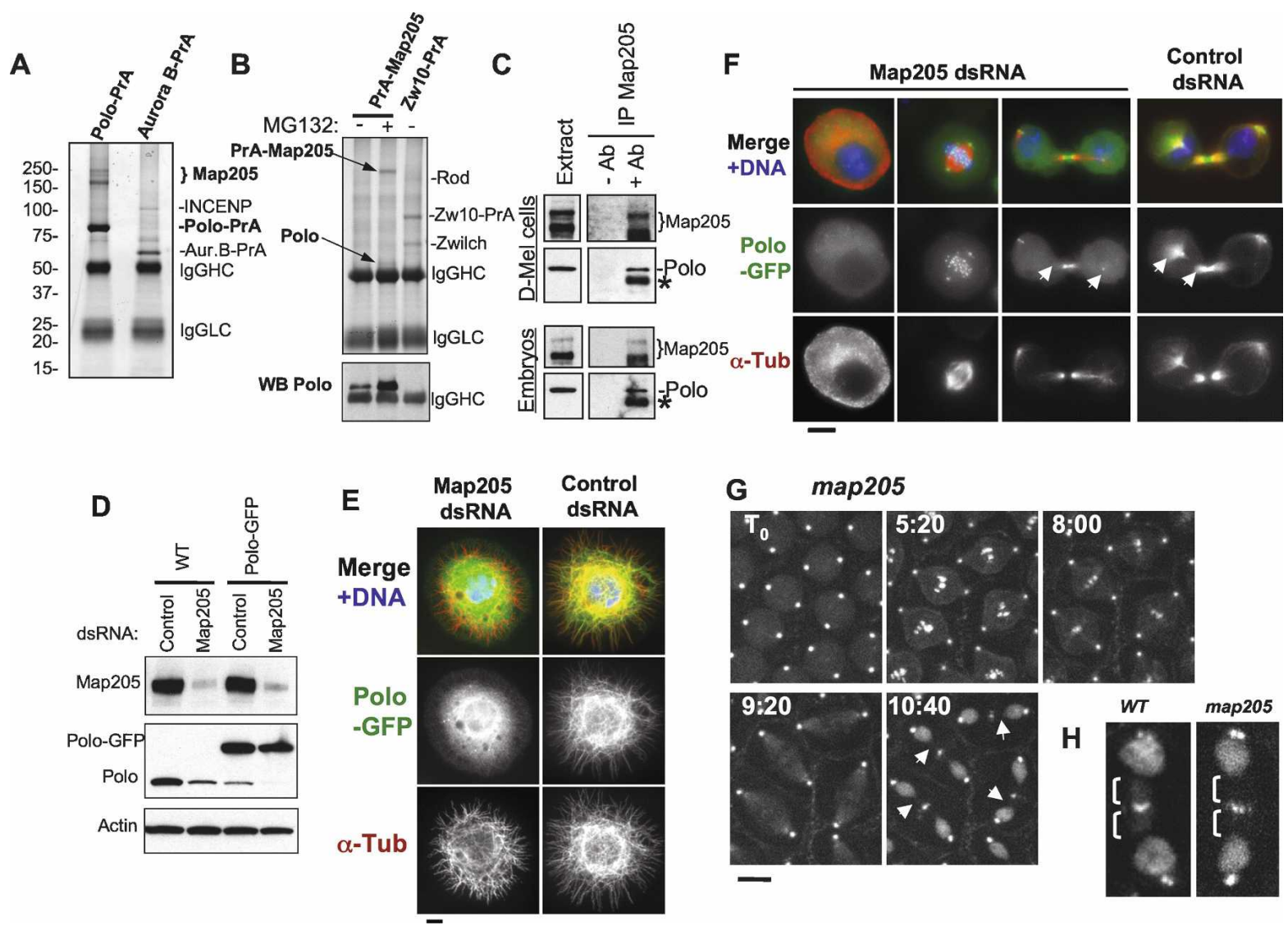

Figure 2. Polo interacts with Map205, and this is required for its localization to MTs. $(A)$ Polo specifically interacts with Map205. Polo-PrA and Aurora B-PrA (control) were purified from stably expressing cell lines, and purified proteins were resolved on SDS-PAGE and stained with Coomassie Blue. The indicated proteins were identified by mass spectrometry. (IgGHC) IgG heavy chain; (IgHLC) IgG light chain. (Left) Molecular mass markers are in kilodaltons (kDa). (B) Reciprocal purification. (Top) PrA-Map205 and Zw10-PrA (control) were purified from stably expressing cell lines, and products were analyzed as in $A$. In one sample, cells were treated overnight with $25 \mu \mathrm{M}$ MG132 to inhibit the proteasome, which results in a stabilization of Map205 (but does not lead to an arrest of the majority of the cells in mitosis). (Bottom) The same purification products were probed for Polo by Western blot. $(C)$ Endogenous Polo and Map205 interact in D-Mel cells and embryos. Polo was coimmunoprecipitated with Map205 using anti-Map205 antibodies from D-Mel cells and from syncytial embryos. As a control, antibodies were not added. ( ${ }^{\star}$ ) Antibodies from the IP cross-reacting in the Polo Western blot. ( $D$ ) Depletion of Map205 by RNAi. D-Mel cells, wild-type or stably expressing Polo-GFP, were treated with dsRNA against Map205 or the bacterial kanamycin-resistance gene (control). Four days later, cells were analyzed by Western blotting against Map205, Polo, and actin (loading control). Note that in addition to the depletion of Map205, Polo levels are also reduced. (E,F) Polo requires Map205 for its localization to MTs. (E) Polo-GFP cells were treated with Map205 dsRNA or control dsRNA (as in D), spread on concanavalin-A, fixed with methanol/formaldehyde, and stained for $\alpha$-tubulin (red) GFP (anti-GFP; green), and DNA (blue). Bar, $5 \mu$ m. $(F)$ Polo-GFP cells were treated with Map205 or control dsRNA (as in $D$ and $E$ ), fixed with formaldehyde, and stained for $\alpha$-tubulin (red) and DNA (blue). (Right) Note that unlike in the control, Polo-GFP fails to colocalize with MTs in cytokinesis after Map205 RNAi (arrows). Also compare with cells in Figure 1B. Bar, $5 \mu \mathrm{m}$. (G) GFP-Polo dynamics in a transgenic syncytial embryo laid by a map205 homozygous null mother (cycle 13) by time lapse (time is shown in minutes:seconds). Note that, unlike in map205 embryos (Fig. 1D), GFP-Polo fails to localize to the MTs of the central spindle during karyokinesis in map205-null embryos (arrows). Bar, $10 \mu \mathrm{m}$. (H) Magnified view of karyokinetic nuclei from wild-type (WT) or map205-null embryos expressing GFP-Polo (taken from G and Fig. 1D). Note that unlike in the wild-type control, GFP-Polo fails to localize to central spindle MTs in the map205-null embryo.

variant that cannot bind Polo, Map205-S283E (see below) (Supplemental Fig. S4). Strikingly, depletion of Map205 also resulted in the loss of either Polo-GFP (Fig. 2E-F) or endogenous Polo (Supplemental Fig. S1) at MTs. MTs also appeared more fragmented and disorganized following Map205 RNAi (Fig. 2E,F). To verify that Map205 was also required to localize Polo on MTs in the fly, we introduced a GFP-Polo transgene in map205-null flies. In embryos laid by map205 homozygous null mothers,
GFP-Polo was no longer visible on the MTs of the central spindle in karyokinesis (cf. Figs. 2G and 1D, 2H). However, Polo-GFP was still able to localize to centrosomes, kinetochores, and the spindle midzone in the absence of Map205 (Fig. 2F-H). Polo localization to the cytokinetic midbody has been previously shown to depend on another MT-associated protein, Feo/PRC1 (D'Avino et al. 2007; Neef et al. 2007), and is therefore distinct from the pool of Polo bound to Map205. Together these results 
demonstrate that Polo's recruitment to MTs in interphase and cytokinesis is strongly dependent on an interaction with Map205.

\section{The Polo-Box Domain is required but not sufficient} for Polo to interact with Map205

Plks stably interact with their targets via the Polo-Box Domain (Elia et al. 2003a,b; Lowery et al. 2004). To test if the PBD of Polo was required for its interaction with Map205 and localization to MTs, we expressed PBD-mutant forms of Polo in fusion with either PrA or GFP. Amino acid substitutions in conserved residues have been shown to disable the PBD of hPlk1 (Seong et al. 2002). We introduced the equivalent mutations in Drosophila Polo (W395F, V396A, and L408A = Polo-WVL) (Fig. 3A). This mutant form of Polo (or a truncated form of Polo removing the PBD) failed to copurify Map205 (Fig. 3B; data not shown) and to localize to centrosomes, kinetochores, the central spindle, or interphase MTs (Fig. 3C,D; data not shown). Even the V396A substitution alone largely abolished the interaction of Polo with Map205 (Fig. 3B) and its localization to MTs (data not shown). Therefore, a functional PBD is required for Polo to interact with Map205 and for MT localization. However, in a copurification test performed using D-Mel cells expressing tagged proteins, we found that the PBD along with the linker separating it from the kinase domain (amino acids 298-576) was not sufficient for this interaction (Supplemental Fig. S5). Altogether, these results strongly suggest that the interaction of Polo with Map205 requires the PBD as well as some structural elements of the kinase domain of Polo.

\section{Polo binding does not require phosphopriming of Map205}

In order to map the binding site of Polo in Map205, we first generated truncations of Map205 fused with PrA and used them in a copurification assay probing for Polo. The robust copurification of PrA-Map205 with endogenous Polo (Fig. 2B) was disrupted by truncations removing a region comprised between amino acid residues 254 and 400 of Map205. Conversely, residues 254-416 were sufficient to interact strongly with Polo (Fig. 4A-C). Several reports suggested that Plks require the PBD to bind to a $\mathrm{S}-(\mathrm{pS} / \mathrm{pT})$ motif on their targets (see Introduction). Residues 254-416 contain only one such potential SS or ST motif (S381-S382) (Fig. 4B), which if phosphorylated at the second residue would correspond to the known Polo kinase-binding motif. We therefore replaced the serine residue with an alanine residue (S382A). Surprisingly, PrA-Map205(254-416)-S382A was still able to bind Polo just as strongly as the wild-type equivalent (Fig. 4C). To our knowledge, this is the first demonstration of an interaction involving Polo (or any Plk) that
A

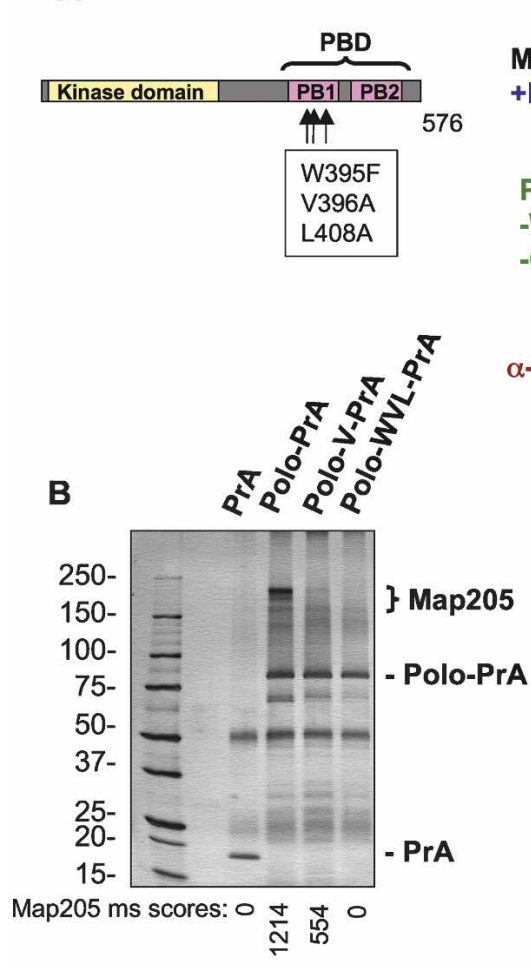

C
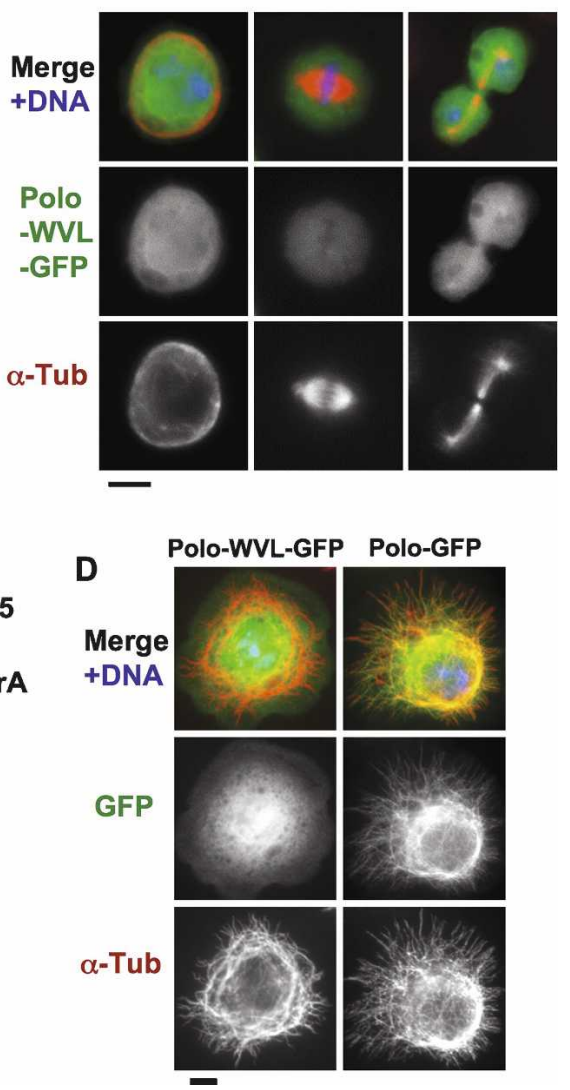

Figure 3. Polo requires a functional Polo-Box Domain to interact with Map205 and to localize to MTs. (A) Amino acid substitutions introduced in Polo to disrupt the Polo-Box Domain (PBD). The kinase domain is in yellow, and the two Polo Boxes (PB1 and PB2) are in pink. (B) A functional Polo-Box Domain is required for Polo to interact with Map205. Stable cell lines expressing Polowt, Polo-V (V396A), Polo-WVL (W395F, V396A, and L408A) in fusion with PrA, or PrA alone were used in affinity purifications. Purified proteins are shown on a Coomassie Blue-stained gel. Each purification product was assayed for the presence of Map205 in the area defined by the bracket. Mascot scores (see Materials and Methods) for Map205 are shown under the gel image. (IgGHC) IgG heavy chain; (IgHLC) IgG light chain. (Left) Molecular mass markers are in kilodaltons ( $\mathrm{kDa})$. $(C, D)$ A functional Polo-Box Domain is required for Polo to localize to MTs. $(C)$ Cells stably expressing Polo-WVL in fusion with GFP (green) were fixed with formaldehyde and stained for $\alpha$ tubulin (red) and DNA (blue). Compare with Figure 1B. Note that Polo-WVL-GFP fails to localize to MTs, kinetochores, centrosomes, and the midbody. Bar, $5 \mu \mathrm{m}$. (D) Cells stably expressing PoloGFP or Polo-WVL-GFP were plated on concanavalin-A, fixed with methanol/formaldehyde, and stained for GFP (anti-GFP; green), $\alpha$-tubulin (red), and DNA (blue). Bar, $5 \mu \mathrm{m}$. 


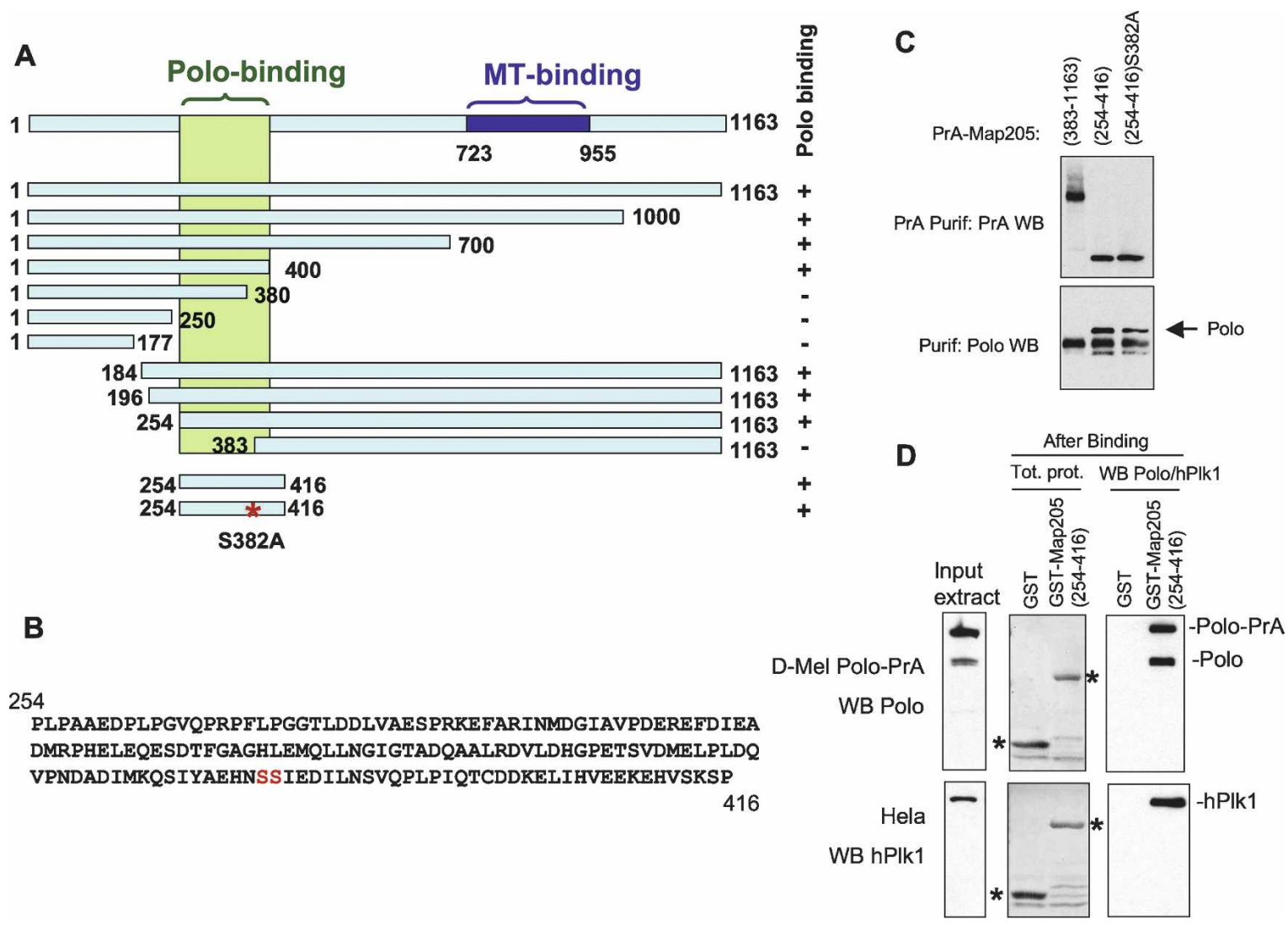

Figure 4. Polo binding to Map205 does not require phosphopriming. (A) Summary of a truncation analysis defining a small region of Map205 necessary and sufficient for Polo binding. The assay used is as in $C$ and Figure $2 \mathrm{~B}$. Polo binds to the region in green. The MT-binding region (blue) was defined elsewhere (Irminger-Finger et al. 1990). (B) Sequence of a small segment of Map205 (amino acids 254-416) sufficient for binding Polo. Note that it contains only one potential canonical PBD-binding motif (SS or ST) at S381-S382. (C) Map205 does not require phosphopriming at a canonical PBD-binding motif (S-pS or S-pT) for Polo binding. Cells transiently expressing the indicated Map205 mutants in N-terminal fusion with Protein A were used in Protein A affinity purification, and the products were probed for Protein A and Polo. Note that Map205(254-416)-S382A can still interact with Polo. (D) Map205 does not require phosphorylation to interact with Drosophila Polo or human Plk1. GST-Map205(254-416) or GST alone (control) were expressed in bacteria, purified (asterisks), and subjected to a Polo or hPlk1-binding assay using D-Mel (stably expressing Polo-PrA) or HeLa cell extracts, respectively. Purification products were analyzed by Western blotting for Polo (top) or hPlk1 (bottom).

does not require a $\mathrm{S}-(\mathrm{pS} / \mathrm{pT})$ phosophopriming motif in vivo. However, the possibility remained that phosphopriming for Polo binding could still occur at a noncanonical site on Map205. To investigate this possibility, we expressed Map205(254-416) in fusion with GST in Escherichia coli. Because bacteria possess little kinase activity, and as their kinases differ strongly from those of eukaryotic cells, Map205(254-416) expressed in bacteria should not be phosphoprimed for Polo binding. We incubated GST-Map205(254-416) beads with a Drosophila cell extract at $4^{\circ} \mathrm{C}$ and probed for Polo binding by Western blotting. Strong and specific binding of Polo was detected in this assay (Fig. 4D). Taken together, these results indicate that the interaction between Polo and Map205 does not require any phosphopriming.

We also found that hPlkl was able to interact strongly with bacterially expressed Map205 in the same assay, using a HeLa cell extract, and independently of priming phosphorylation (Fig. 4D). This is consistent with the previously reported ability of GFP-hPlk1 to localize to the central spindle during karyokinesis when heterologously expressed in Drosophila syncytial embryos (Pearson et al. 2006). In the case of Drosophila GFP-Polo, this localization requires Map205 (Fig. 2G,H), and it seems reasonable to assume that the localization of hPlk1 to the central spindle observed in Drosophila embryos is also mediated by its interaction with Map205. The phosphopriming-independent interaction between Polo or hPlk1 and Map205 reinforces the emerging idea that Plks can engage in such interactions (Garcia-Alvarez et al. 2007).

\section{Phosphorylation of Map205 by Cdk1 disrupts its interaction with Polo}

Because we initially suspected that Polo's interaction with Map205 would require phosphopriming, we mapped phosphorylation sites on Map205 by mass spectrometry (Supplemental Fig. S6). We identified four sites: S283, S679, S690, and S852. These are novel sites that 
were not reported in a previous study that identified phosphorylation sites in the Drosophila proteome, including other sites in Map205 (Bodenmiller et al. 2007). One of the sites we identified, S283 is located in the region of Map205 that mediates Polo binding (Figs. 4A, $5 \mathrm{~A})$. We noticed that S283 lies in a perfect consensus motif for phosphorylation by cyclin-dependent kinases (SPRK; the ideal motif is S/T-P-X-K/R) (Nigg 1993). Indeed, human cyclin B-Cdk1 was able to strongly phosphorylate Map205(254-416) in vitro, but not the equivalent S283A mutant form (Fig. 5B). Phosphorylation of Map205(254-416) by cyclin B-Cdk1 or substitution at S283 with a negatively charged, phospho-mimic aspartic acid residue (S283D) reduced its affinity for Polo in vitro
(Fig. 5C). The phospho-mimic mutations S283D and S283E also abolished the binding of Polo to Map205 in vivo, while the S283A mutation had no detectable effect (Fig. 5D). To test the effect of phosphorylation on Polo's localization in vivo, we made stable cell lines expressing either Map205-Myc or the phospho-mimic mutants Map205-S283D-Myc and Map205-S283E-Myc. All fusion proteins were still able to localize to MTs (Supplemental Fig. S7). We then depleted the endogenous Map205 in those cell lines using dsRNA against an untranslated region (UTR) of the transcript (Fig. 5E). While expression of wild-type Map205-Myc did not affect the colocalization of Polo with $\alpha$-tubulin in interphase and cytokinesis, the S283D mutant disrupted this localization, leaving Polo

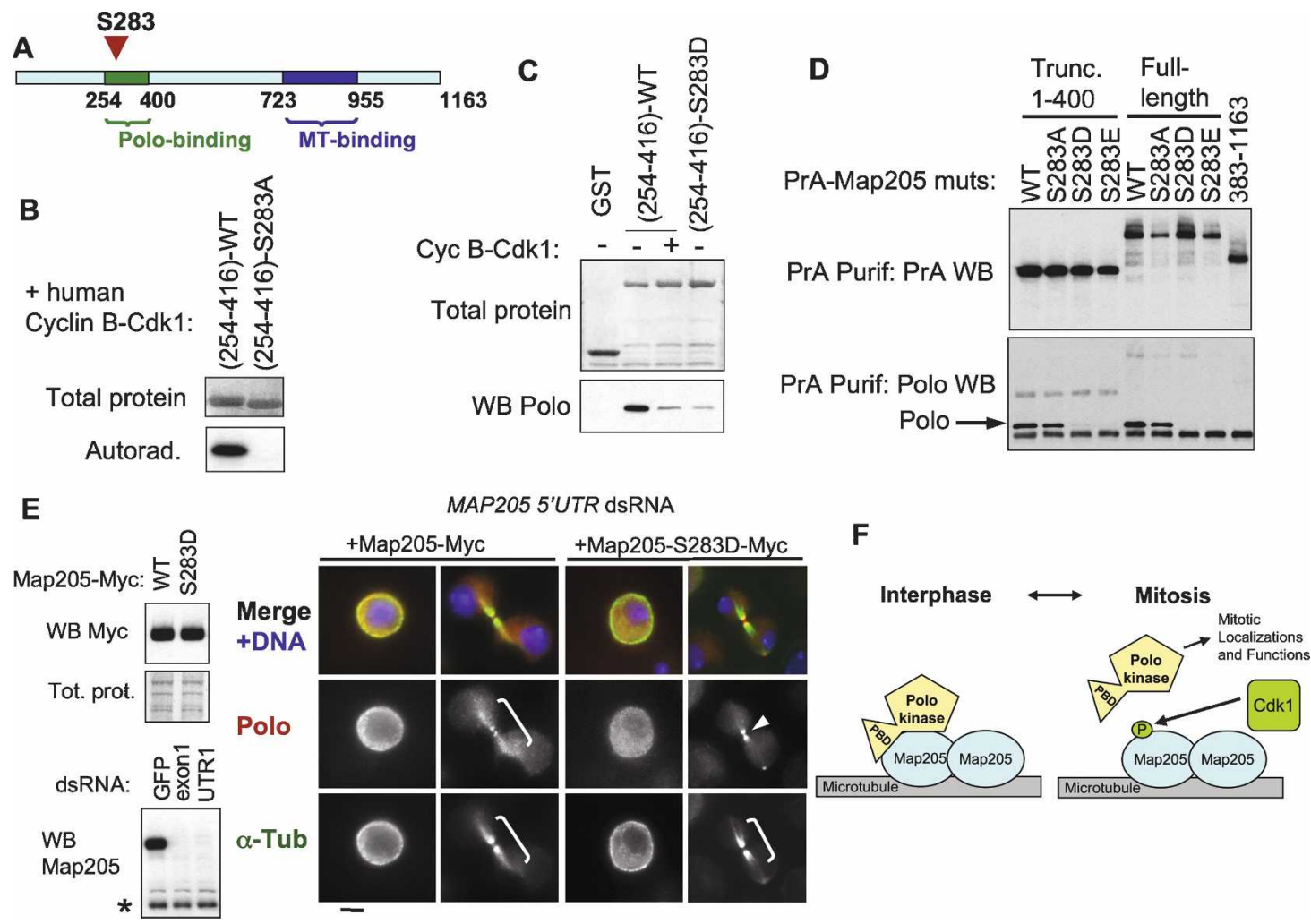

Figure 5. Map205 phosphorylation at Cdk1 site Ser 283 prevents interaction with Polo. (A) Map205 is phosphorylated at Ser 283, inside the Polo-binding region in vivo (for details, see Supplemental Figure S6). (B) Ser 283 of Map205 is a target of cyclin B-Cdk1 in vitro. Map205(254-416)-wt or S283A was generated using bacterial expression and subjected to a kinase assay using human cyclin B-Cdk1. The total protein (amido black staining) and the autoradiograph are shown. (C) Phosphorylation of Map205(254-416) by cyclin B-Cdk1 or phosphomimicking at Ser 283 inhibits the interaction with Polo in vitro. The indicated proteins were treated or not with cyclin B-Cdk1 before being subjected to a Polo-binding assay as in Figure 4D. (D) Mimicking phosphorylation at Ser 283 of Map205 abolishes its interaction with Polo in vivo. Cells transiently expressing the indicated proteins (full-length or truncated Map205) were used in Protein A affinity purification, and the products were probed for PrA and Polo. Note that the phosphomimicking S283D and S283E mutations abolish the interaction with Polo. (E) Phosphomimicking phosphorylation at S283 disrupts the localization of Polo on MTs. (Top left) Stable cell lines expressing Map205-Myc (wild type and S283D) were created and expressed at similar levels. RNAi targeting an untranslated region of the endogenous MAP205 transcript was used to deplete endogenous Map205. (Bottom left) The Western blot shown is from cells not transfected with Map205 cDNA, for clarity. Cells were then stained for Polo (red), $\alpha$-Tubulin (green), and DNA (blue). Note that Polo is present on MTs (brackets) when Map205-Myc wild type, but not S283D is expressed. Polo is sometimes detected at the midbody (arrow) even when it does not localize to MTs. Bar, $5 \mu \mathrm{m}$. (F) Model for the regulation of Polo localization by Map205 and Cdk1. In interphase, Polo is sequestered to MTs via an interaction with Map205 that depends on both the $\mathrm{PBD}$ and the kinase domain of Polo, but that does not require priming phosphorylation. At mitotic entry, Cdk1 phosphorylates Map205, which releases Polo from Map205 and MTs. Polo is then free to translocate to its mitotic localizations, where it functions to promote proper mitosis. In late mitosis, Cdk1 becomes inactivated, and Polo returns to MTs in cytokinesis. 
only in the cytoplasm and on the midbody (Fig. 5E; midbody staining is not always observed). Similar results were obtained when the S283E mutant was expressed (data not shown). As shown above, Polo localization to MTs is strong in interphase and cytokinesis, when cyclin B-Cdk1 activity is low, and is almost lost completely in early to mid-mitosis, when cyclin B-Cdk1 is most active. Thus, together, our results strongly suggest that cyclin B-Cdk1 phosphorylation of Map205 in mitosis disrupts the interaction between Polo and Map205 until Cdk1 inactivation at anaphase onset. Therefore, Cdk1 activation at mitotic entry is poised to promote the release of Polo from Map205 (Fig. 5F), enabling Polo to concentrate on kinetochores, centrosomes, and centromeres, where it can conduct its essential mitotic functions (van de Weerdt and Medema 2006). Inactivation of cyclin B-Cdk1 in late mitosis would allow Polo to bind Map205 again.

\section{Map205 contributes to the regulation of Polo in vivo}

To explore the function of Polo interaction with Map205 in vivo, we generated transgenic fly lines expressing Myc-tagged Map205, either wild type or mutated at S283 (the Cdk1 phosphorylation site), under the control of the $U A S p$ promoter. We induced moderate overexpression of the Map205 transgenes in the female germline using the maternal $\alpha$-Tubulin Gal-4 driver, allowing for a maternal contribution to the early embryo. Females overexpressing wild-type Map205 and heterozygous for the polo ${ }^{11}$-null allele (Archambault et al. 2007) or a small deficiency uncovering polo, laid embryos, but a majority of them failed to hatch (Fig. 6A; Supplemental Fig. S8). This effect was dependent on the ability of Map205 to bind Polo because overexpression of the Map205-S283E mutant, which fails to interact with Polo (Fig. 5), did not cause a failure of embryos to hatch. As expected, the S283A mutant, which presumably retains the ability to bind Polo even when Cdk1 is active (Fig. 5), caused an even more severe hatching failure than Map205-wt (Fig. 6A; Supplemental Fig. S8). Moreover, this effect was dependent on a reduced gene dosage of Polo because embryos derived from polo ${ }^{+}$females could hatch normally even when Map205 was overexpressed (Fig. 6A). To investigate potential effects on mitotic divisions, we examined syncytial embryos at the early blastoderm stage (cycles 10-13) by immunofluorescence. Embryos produced by polo heterozygous females overexpressing Map205 displayed a very high percentage of mitoses in which a single centrosome detached from the nuclear envelope in prophase (Fig. 6B,C). This defect is extremely rare in wild-type embryos but was, instead, significantly increased in syncytial blastoderm embryos derived from polo ${ }^{11}$ heterozygous females (Fig. 6B; Archambault et al. 2007). Penetrant single centrosome detachment from nuclei in prophase in early blastoderm embryos was recently found to be the prevalent and initial defect occurring when the ratio of Greatwall:Polo activity was increased, and there too this was associated with a failure in embryonic development (Archambault et al. 2007).
This study indicated that Greatwall could act to downregulate Polo or a Polo-dependent pathway required for the cohesion between centrosomes and the nuclear envelope (Archambault et al. 2007). In both cases, detached centrosomes were also detected in later mitotic stages, leading to mono-astral spindles (Fig. 6B,C; Archambault et al. 2007). However, the percentage of nuclei showing centrosome detachment in prometaphase to telophase was lower than in prophase (Fig. 6B). This is consistent with time-lapse studies showing that detached centrosomes can be recaptured by growing spindles in prometaphase (Archambault et al. 2007). As expected, the centrosome detachment defects were also dependent on the ability of Map205 to interact with Polo (Fig. 6D); overexpression of Map205-S283A induced more centrosome detachment than Map205-wt (86\% vs. 64\%, respectively), while overexpression of Map205-S283E induced considerably fewer defects $(13 \%$, similar to polo $11 /+$ alone). Our results strongly suggest that Map205 is able to inhibit Polo's cellular activity in vivo by direct binding. We note that such an inhibition does not necessarily require direct inhibition of the kinase domain. This presumably imposes a requirement for Cdk1-dependent release of Polo from Map205 at mitotic entry.

In order to assess if Map205 contributes to regulate Polo in vivo, we tested if the removal of Map205 would rescue or enhance phenotypes observed in the context of a partial loss of Polo function. Although polo heterozygous females laid embryos that develop until adulthood, these embryos show a low but significant frequency of single centrosome detachment in prophase nuclei of the syncytial blastoderm (Fig. 6B). By scanning deeper into early syncytial embryos, we were able to examine the preblastoderm nuclei in cycles $8-9$ and found that polo heterozygous-derived embryos had a much higher incidence of single centrosome detachment in prophase at that stage, which was almost never observed in wildtype-derived embryos (Fig. 6E; Supplemental Fig. S9). This single centrosome detachment did not cause embryonic lethality because nuclei kept the ability to migrate to the cortex, probably because they always remained attached to one centrosome (we observed no gross increase in the number of yolk nuclei) (data not shown) and because the detached centrosome could be recaptured. We tested how this polo-dependent defect was affected by deletion of map205. Western blot analysis revealed that deletion of map205 did not destabilize Polo in the embryo (Fig. 6F) as it did in cultured cells (Fig. 2D); Polo degradation likely requires Fizzy related, which is absent in embryos (see Discussion). Strikingly, the frequency of single centrosome detachment observed in heterozygous polo-derived embryos was suppressed following deletion of map205 (Fig. 6E; sample images are shown in Supplemental Fig. S9). This rescue was observed in both preblastoderm (from 69\% to 36\% nuclei with centrosome detachment) and syncytial blastoderm embryos (from $16 \%$ to $7.5 \%$ ). We therefore conclude that endogenous Map205 partially restrains the cellular activity of Polo in syncytial embryos. 
A

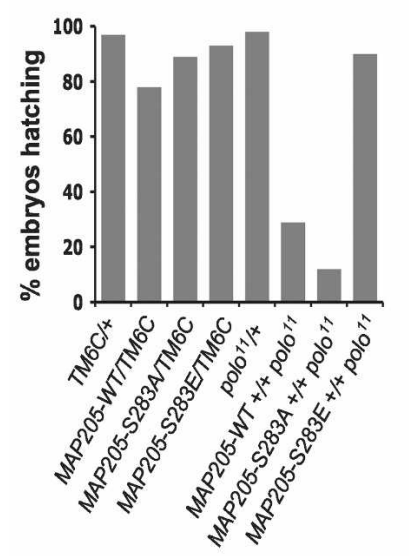

B

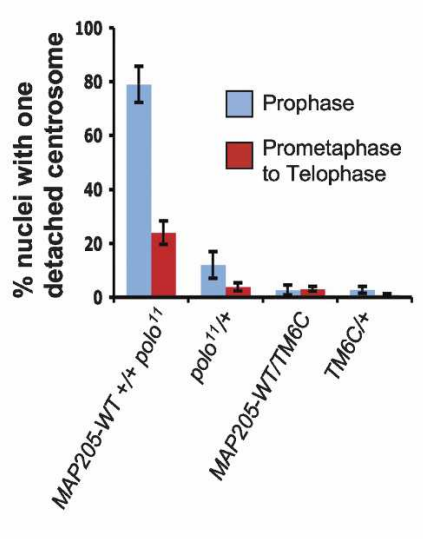

D

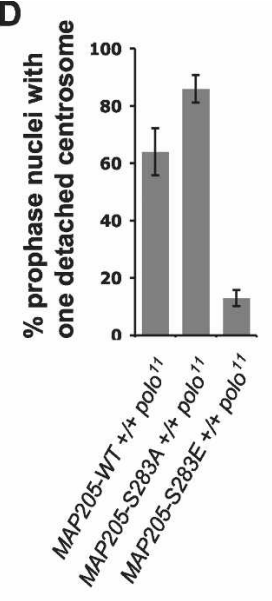

C

$T M 6 C /+$
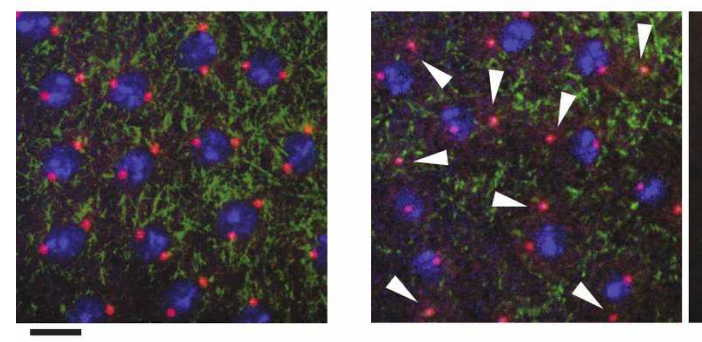

E

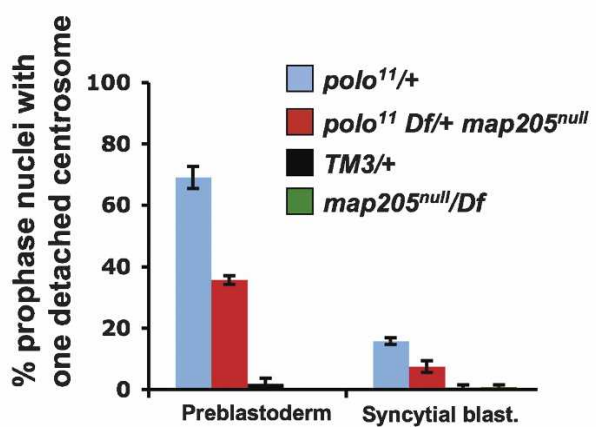

+ polo 11
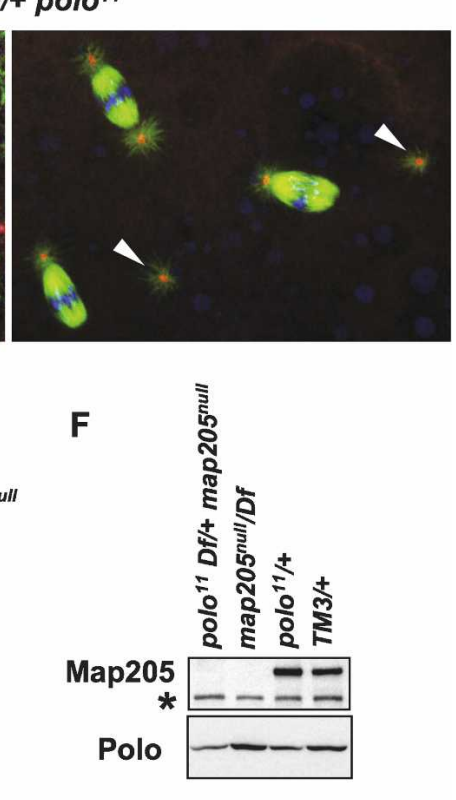

Figure 6. Map205 contributes to the regulation of Polo in vivo. $(A-C)$ Overexpression of Map205 enhances Polo-dependent defects. (A) Percentage of hatching embryos produced by females of the indicated genotypes. All genotypes included one copy of the Maternal $\alpha$-Tubulin Gal4 driver (not shown). TM6C is a balancer chromosome. Map205 transgenes were under the control of the UASp promoter. Transgenic lines were selected for equal and moderate overexpression levels of Map205 variants (Supplemental Fig. S8). At least 1000 embryos laid by 12 females over $3 \mathrm{~d}$ were scored for each genotype. (B) Percentage of nuclei showing single centrosome detachment in syncytial blastoderm embryos (cycles 10-13) produced by females of the indicated genotypes (as in $A$, separate experiment). Between five and 10 embryos were scored for each entry $( \pm \mathrm{SEM}) .(C$, right $)$ Examples of single centrosome detachment (arrows) observed in prophase and in metaphase (genotypes are as in $A$ and $B$ ). The metaphase spindles on the bottom and on the right show typical single centrosome detachment, while the spindle on the top appears normal. (Left) Image taken from a control embryo where both centrosomes remain normally tethered to the nuclear envelope in prophase. Stainings are $\alpha$-Tubulin (green), $\gamma$-Tubulin (red), and DNA (blue). Bar, $10 \mu \mathrm{m}$. (D) Percentage of prophase nuclei showing single centrosome detachment in syncytial blastoderm embryos (cycles 1013) produced by females of the indicated genotype (as in $A$ and $B$, separate experiment). Ten embryos were scored for each entry $( \pm$ SEM). (E) Deletion of Map205 partially rescues the Polo-dependent centrosome detachment. Percentage of prophase nuclei showing single centrosome detachment in preblastoderm (cycles 8-9) and early blasto-

derm (cycles 10-11) embryos produced by females of the indicated genotypes. Note that single centrosome detachment is frequent in embryos laid by polo $11 /+$ females and that this defect is partially rescued by deletion of map205. Between five and 10 embryos were scored for each entry $( \pm$ SEM). Refer to Supplemental Figure S9 for sample images. $(F)$ Western blot from embryos laid by females of the indicated genotypes (0-3 h collection; as in E). (Top) Map205 detection. ( $\left.{ }^{\star}\right)$ Cross-reacting band serving as a loading control. (Bottom) Polo detection. Note that the absence of Map205 does not correlate with a lower amount of Polo in embryos (unlike in cultured cells).

\section{Discussion}

How Plks are controlled in space and time during the cell cycle is not completely understood, despite three known levels of control. First, hPlk1 has been shown to be ubiquitinated and degraded by the proteasome in mammalian cells (Ferris et al. 1998; Lindon and Pines 2004). However, the importance and extent of conservation of this mechanism are still to be determined. For example, although the destruction box identified in hPlk1 appears to be conserved in Drosophila Polo (Lindon and Pines 2004), Polo does not disappear (or even decrease significantly in abundance) between mitosis and interphase (Fig. 1; Supplemental Fig. S1). Moreover, even in mammalian cells, a substantial fraction of the hPlk1 pool re- mains in G1 (Lindon and Pines 2004). Second, human Plk1 and Xenopus Plx1 are activated in mitosis by phosphorylation at a threonine residue in the T-loop (T210 in hPlk1, T201 in Plx1) (Qian et al. 1999; Kelm et al. 2002). As this site is conserved, this likely acts as a universal activation mechanism. In human cells, Aurora A and its adaptor protein Bora have been shown recently to activate hPlk1 by phosphorylation at T210 (Macurek et al. 2008; Seki et al. 2008). A third control mechanism lies in docking of Plks to prephosphorylated targets using the PBD. This provides an efficient way to target Plks to their mitotic targets (Elia et al. 2003b; Lowery et al. 2004). It has also been recently shown that PBD-dependent sequestration of Polo to a strong binding partner, a female germline-specific protein named Matrimony 
(Mtrm), can keep this kinase inactive in meiotic prophase I (Xiang et al. 2007). Polo later overcomes this inhibition, and is thought to trigger germinal vesicle breakdown (Xiang et al. 2007). This interaction depends on phosphopriming of Mtrm. In contrast, we now describe a mechanism for regulating Polo through its cell cycle-regulated sequestration to MTs by strong binding to a MT-associated protein in a manner that is not primed by phosphorylation but nonetheless requires the PBD.

Our results shed new light on how Drosophila Polo is regulated at mitotic entry and exit. Based on the results shown above, we suggest a model whereby Polo is sequestered on MTs (and can be stabilized) in interphase by interacting with Map205, and where this interaction is negatively regulated by Cdk1 activity in early mitosis (Fig. 5F). Although Polo kinases have been reported to interact with tubulins in vitro (Feng et al. 1999; Neef et al. 2003), this does not appear to provide an efficient mechanism of Polo targeting to MTs, which is instead strongly dependent on Map205. The cellular concentration of Map205 is very similar or possibly higher than that of Polo (Supplemental Fig. S10), consistent with a role for Map205 in sequestering Polo. Such sequestration of Polo onto MTs could serve to store and stabilize the kinase in an innocuous location, until needed at mitotic entry.

While we show that phosphorylation of Map205 at S283 is sufficient to block its interaction with Polo and the localization of Polo to mitotic MTs, we do not know if this phosphorylation event is necessary for inhibiting the targeting of Polo to MTs. For example, phosphorylation of Map205 at other sites may also contribute to negatively regulate the interaction. We have not yet rigorously tested this possibility. However, the fact that overexpression of Map205-S283A in embryos enhances polo-dependent defects more strongly than overexpression of Map205-wt suggests that phosphorylation of Map205 at S283 is necessary for full inhibition of the Map205-Polo interaction in vivo.

We showed that the single centrosome detachment from prophase nuclei observed in polo mutants is enhanced by overexpression of Map205 and suppressed by the loss of Map205 function. Centrosome detachment appeared more sensitive to a reduction in Polo activity in the preblastoderm than in the early blastoderm embryo. We speculate that this may be due to a difference in the forces that the centrosome-nuclear envelope linkage must withstand at those different stages. The organismal and cellular phenotypes observed in polo mutants after Map205 overexpression were dependent on the ability of Map205 to interact with Polo and identical to those caused by an increase in the activity of Greatwall, a recently identified antagonist of Polo (Archambault et al. 2007). Importantly, the suppression of the polo-dependent centrosome-detachment phenotype by deletion of map205 is consistent with the notion that endogenous Map205 functions to regulate Polo in vivo. Sequestering of Polo by Map205 could prevent it from accessing its targets prematurely on centrosomes and kinetochores, whether or not Polo's kinase activity is inhibited at the structural level. Our antibodies against Polo and Map205 did not allow visualization of the endogenous proteins in the embryo for technical reasons, but the genetic, functional, and biochemical results obtained in this system support the model presented here. Exploring what other tissues rely on this Polo-control pathway should be the subject of future studies.

In addition, the interaction with Map205 appears to stabilize Polo (see below). In this way, the Map205-Polo complex on MTs (or even if partly present as a cytosolic pool) could constitute a reservoir of Polo, making Polo available for rapid, Cdk1-triggered mobilization at mitotic entry, and resequestration at mitotic exit. The prevention of hPlk1 from binding PRC-1 by Cdk1-dependent phosphorylation was recently identified as a mechanism for timing hPlk1 localization to the midbody (Neef et al. 2007). Our findings augment the general model whereby the choice of Polo kinase binding partners is controlled by Cdk1 activity during the cell cycle (Neef et al. 2007). Our model does not exclude the possibility that Polo is targeted to MTs for another function. For example, Polo could regulate Map205. Indeed, we found that Polo can phosphorylate Map205 in vitro (Supplemental Fig. S11), and the physiological relevance of this phosphorylation remains to be investigated. Additionally, targeting of Polo to MTs could facilitate its regulation of motor proteins (Neef et al. 2003) or other MTassociated proteins with roles in interphase or cytokinesis.

We identified orthologs of Map205 in the 12 sequenced Drosophila genomes. An alignment between these protein sequences (Supplemental Fig. S12) shows that the N-terminal Polo-binding region of Map205 (within amino acids 254-416 in Drosophila melanogaster) is very well conserved. Importantly, the Cdk1 phosphorylation site (S283) and the sequence immediately surrounding it (VAESPRK) are perfectly conserved between all 12 species. In fact, the Polo-binding region appears to be even better conserved than the C-terminal MT-binding region (amino acids 723-955 in D. melanogaster). This suggests to us that the Polo-binding function of Map205 may be at least as important as its MT-binding function, and that the molecular mechanisms reported here are likely to be conserved between species.

RNAi depletion of Map205 in cultured Drosophila cells leads to perturbations in the cell cycle profile, including an increase in the percentage of cells in mitosis, and more specifically in prometaphase. This may be a consequence of the Polo destabilization (Fig. 2D; Supplemental Fig. S4), since partial loss of Polo function also results in an accumulation of cells in prometaphase. By replacing endogenous Map205 with a form of Map205 that cannot bind Polo (Map205-S283E), we verified that the destabilization of Polo following Map205 depletion in D-Mel cells results from the loss of interaction between Polo and Map205 and not from other perturbations arising from the loss of Map205 (Supplemental Fig. S4). In contrast, removal of Map205 from the early embryo did not result in a destabilization of Polo (Fig. 6F). 
Budding yeast Cdc5 and human Plk1, both orthologs of Polo, undergo cell cycle-regulated degradation that is thought to depend on Cdh1 (Lindon and Pines 2004; Visintin et al. 2008). If Drosophila Polo is degraded by the same pathway, our results can be explained by the absence of Fizzy related (Drosophila Cdh1) in the early embryo (Sigrist and Lehner 1997).

Our experiments reveal that Map205 can strongly alter the subcellular localization of Polo (by targeting Polo to MTs), stabilize Polo in D-Mel cells, and inhibit Polo's cellular activity in the embryo (probably by sequestering of the enzyme away from its substrates). Yet, despite the ability of Map205 depletion to delay progression through prometaphase in cultured cells, map205-null flies are viable and fertile (Pereira et al. 1992), and we did not detect obvious mitotic defects in their embryos or dividing neuroblasts (data not shown). Because of the existence of at least three more, partially overlapping levels of control of Polo activity-proteolysis, activating phosphorylation, phosphopriming of Plk docking-it is difficult to assess the consequences of a total failure to down-regulate and stabilize Plks in interphase. Other mechanisms regulating Polo may compensate for the loss of the Map205-dependent pathway in most tissues. Indeed, one would need to combine disruptions of the multiple regulatory mechanisms for Polo or hPlk1 to determine the effects of a full failure to down-regulate or up-regulate these kinases in the cell cycle, and these experiments may become possible as we continue to dissect the pathways controlling these kinases. The existence of multiple, partially redundant control systems is seen for other cell cycle regulators with functions at key stages of cell cycle progression (for example, Cdk1, the APC, or the prereplicative complex). Such redundancy in regulatory mechanisms can provide robustness to the system that can become crucial in situations of stress.

We showed that the Polo-Map205 interaction does not depend on priming phosphorylation. Interestingly, the PBD is essential for this interaction, but it is not sufficient, and therefore some elements of the kinase domain appear to be also needed. This is the first time that such requirements have been demonstrated for a Plk interaction in vivo. This phosphorylation-independent mode of interaction is well suited to control Polo in interphase, when cellular kinase activities are typically low. Since hPlk1, like Drosophila Polo, can also bind Map205, this mode of interaction is likely to be conserved for other Plks and could potentially involve other substrates. It was recently shown that the PBD of hPlk1 is capable of interacting with Cdc25C peptides with similar affinities for the phospho- and nonphospho forms in vitro (GarciaAlvarez et al. 2007). The full biological significance of these modes of interaction and how they affect the activity of the kinase should become clearer as the molecular details of more Plk-target interactions are investigated.

The strong interaction between hPlk1 and Map205 in vitro also raises the possibility that the type of regulatory mechanism identified here may be conserved beyond the Drosophila genus. We note that there is relatively poor sequence conservation between Map205 and its human ortholog, Map4, (our unpublished observations) but this is not unusual among MT-associated proteins. The relative importance of the different mechanisms for regulating the location and activity of Plks-activating phosphorylation, proteolysis, and protein binding-will almost certainly depend on the organism and may even vary between cell and tissue types. As different types of cancer cells may undergo deregulation of different hPlk1 control mechanisms, it will be of considerable interest to further explore the relative contributions of these alternative mechanisms for regulating hPlk1 in different disease situations.

\section{Materials and methods}

\section{DNA constructs}

Drosophila expression vectors were made using the Gateway technology (Invitrogen). The Polo, Aurora B, Zw10, and Map205 genes (as well as truncations of Map205 and Polo) were cloned by recombination of PCR products into the pDONR221 $\lambda$ entry vector (Invitrogen). The same genes were then recombined into the relevant destination vectors for expression in fusion with Protein $\mathrm{A}, \mathrm{GFP}$, or $\mathrm{Myc}$, at the $\mathrm{N}$ or $\mathrm{C}$ terminus, and under the control of the Actin 5C, UASp, or MT (Metallothionein) promoter. Amino acid substitution mutants were made using QuickChange (Stratagene). Bacterial expression constructs for Map205 fragments in N-terminal fusion with GST-TEV were made by inserting MAP205 cDNA into $p$ GEX4T-GST-TEV.

\section{Cell culture, cell lines, and RNAi}

D-Mel cells (Invitrogen) were cultured in SFM medium (Invitrogen) supplemented with $1 \mathrm{mM}$ glutamine, penicillin, and streptomycin. Transfections and stable cell line selection were done as described (D'Avino et al. 2006). Transiently transfected cells were analyzed 3 d later. For RNAi, $3 \times 10^{6}$ cells were transfected with $30 \mu \mathrm{g}$ of dsRNA and Transfast (Promega) and allowed to grow in SFM for 3-4 d before being assayed. dsRNAs were synthesized from PCR products; oligonucleotide sequences are available upon request.

\section{Fly genetics}

The GFP-Polo X-chromosome insertion used (Moutinho-Santos et al. 1999) was expressed from the Polo promoter, at levels very similar to endogenous Polo (data not shown). The map205-null third chromosome used was documented elsewhere (Pereira et al. 1992) and consisted in a small deletion removing the genes map205 and modulo, and where modulo was reinserted as a transgene. Transgenic flies for expression of UASp-Map205$M y c$ (wild type and mutants) were created by injections of our plasmids by BestGene, Inc. Insertions on the third chromosome allowing very similar levels of expression were selected for experiments (Supplemental Fig. S8). The deficiencies used were the DrosDel $D f(3 R) E D 6362$ (from John Roote) uncovering map205 and $D f(3 L, 77 A 1-77 D 1)$ (from Adelaide Carpenter), uncovering polo.

\section{Protein affinity purification and immunoprecipitation}

Large-scale Protein A-affinity purifications (Figs. 2A,B, 3B) were performed as described (Chen et al. 2007). Small-scale Protein A-affinity purifications (Figs. 4A,C, 5D; Supplemental Figs. S5, 
S11) followed a similar protocol. Briefly, between $3 \times 10^{7}$ and $1 \times 10^{8}$ cells stably or transiently expressing the Protein A fusion were used as starting materials. Cells were lysed by four cycles of freeze-thaw in lysis buffer $(75 \mathrm{mM}$ K-HEPES at $\mathrm{pH} 7.5$, $150 \mathrm{mM} \mathrm{KCl}, 2 \mathrm{mM} \mathrm{MgCl}$, $2 \mathrm{mM}$ EGTA, 5\% glycerol, $0.1 \%$ NP-40, Complete Protease Inhibitors [Roche]). Lysates were clarified by centrifuging at $20,000 \mathrm{~g}$ for $30 \mathrm{~min}$ in a tabletop centrifuge at $4^{\circ} \mathrm{C}$. Supernatants were incubated for $1-2 \mathrm{~h}$ with Dynabeads M270 Epoxy (Invitrogen) conjugated to whole rabbit IgG (MP Biochemicals). Beads were washed five times with 1 $\mathrm{mL}$ of lysis buffer for 5-10 min. Proteins were eluted from the beads with $0.5 \mathrm{M} \mathrm{NH}_{4} \mathrm{OH}, 0.5 \mathrm{mM}$ EDTA, and desiccated before being redissolved in Laemmli buffer for SDS-PAGE (Sigma). For immunoprecipitations (Fig. 2C) of Map205 from D-Mel cells, extracts were prepared as above, but without glycerol in the lysis buffer. For immunoprecipitations of Map205 from embryos (Fig. 2C), embryos from 0-3-h collections were dechorionated in $50 \%$ bleach, crushed in lysis buffer (without glycerol), and clarified as above. In both cases, clarified lysates were incubated with Map205 antibodies for $2 \mathrm{~h}$ and with Protein Aconjugated DynaBeads (Invitrogen) for a further $2 \mathrm{~h}$, before being washed in lysis buffer as above and eluted in $100 \mathrm{mM}$ glycine (pH 2.5).

\section{Immunofluorescence and Western blotting}

For the experiments presented in Figures 1B, 2F, 3C, and 5E and Supplemental Figures S1, S2, and S3, cells were fixed with formaldehyde and stained as described (Bettencourt-Dias et al. 2004), and the antibodies were rat anti- $\alpha$-Tubulin YL1/2 (Sigma), mouse monoclonal anti-Polo M294, rabbit anti-Map205 (gift from Andrea Pereira), rabbit anti-phospho-H3 (Upstate Biotechnologies), anti-rat-FITC (Jackson), anti-rat-Rhodamine (Jackson), anti-mouse-Rhodamine (Jackson), anti-mouse-FITC, and anti-rabbit-Rhodamine (Jackson). For the experiments presented in Figures 1C, 2E, and 3D and Supplemental Figure S7, cells were plated on concanavalin-A (Rogers et al. 2002) and fixed with $90 \%$ methanol, $3.3 \%$ formaldehyde, $5 \mathrm{mM} \mathrm{NaHCO}_{3}$ for 15 min on dry ice, and then for $12 \mathrm{~min}$ at room temperature. Cells were then washed with PBS and stained as in Bettencourt-Dias et al. (2004); the antibodies were rabbit anti-GFP A6455 (Invitrogen), mouse monoclonal anti- $\alpha$-Tubulin DM1A (Sigma), rabbit anti-Myc (Santa Cruz Biotechnologies), anti-rabbit-Alexa488 (Invitrogen), anti-mouse-Alexa594 (Invitrogen), and anti-rabbitAlexa488 (Invitrogen). Embryos were stained as described (Archambault et al. 2007). Western blotting was done using a standard method, and the antibodies were Protein A: peroxidaseconjugated rabbit IgG (Jackson 011-030-003); Polo: M294 monoclonal; Map205 (gift from Andrea Pereira).

\section{Protein identification and phosphorylation site mapping} by mass spectrometry

Protein identification was performed as described (Chen et al. 2007).

For phosphorylation site mapping, Bands corresponding to potential phosphoproteins were excised from the gel, cut into squares, and subjected to reduction and alkylation with DTT and iodacetamide, respectively. Proteins were digested overnight with trypsin. Reduction, alkylation, and digestion were performed using a MassPrep station (Waters Corporation). Separation of peptides before mass spectrometry was performed by reverse-phase chromatography using an Eksigent NanoLC-1D Plus (Eksigent Technologies) and an LC-Packings (Dionex) C18, $75 \mu \mathrm{M}$ PepMap column. All mass spectrometry experiments were performed using either LTQ or LTQ XL (fitted with an electron transfer dissociation [ETD] [Syka et al. 2004] EI/CI source) instruments (ThermoFisher). The LTQ was operated in a data-dependent neutral loss mode, in which peptide ions were subjected to collision-induced dissociation (CID) to generate sequence-specific fragment ions. The LTQ XL was operated to switch between standard CID and ETD modes in a data-dependent manner. For identification of proteins, the MS/MS data were used to search the mouse NCBI database using the Mascot (Matrix Science) or SEQUEST (ThermoFisher) search engines.

\section{Kinase assays}

For the kinase assay shown in Figure 5B, GST-TEVMap205(254-416)-wt or S283A was expressed in bacteria and purified using Sepharose-4B (Amersham). Proteins were eluted with the TEV protease (Invitrogen). Elution proteins were subjected to a kinase reaction using human cyclin B-Cdk1 (Invitrogen) in $20 \mathrm{mM}$ K-HEPES (pH 7.5), $2 \mathrm{mM} \mathrm{MgCl} 2,1 \mathrm{mM}$ DTT, $500 \mu \mathrm{M}$ ATP, $\left[\gamma^{-32} \mathrm{P}\right] \mathrm{ATP}$, incubating for $20 \mathrm{~min}$ at $30^{\circ} \mathrm{C}$. Reactions were stopped by heating in Laemmli SDS-PAGE sample buffer (Sigma), and reaction products were resolved by SDSPAGE and transferred to nitrocellulose, and membranes were analyzed by autoradiography. For the kinase assay shown in Supplemental Figure S11, protein complexes on beads were directly incubated in $20 \mathrm{mM}$ K-HEPES (pH 7.5), $2 \mathrm{mM} \mathrm{MgCl}_{2}, 1$ mM DTT, $500 \mu \mathrm{M}$ ATP, and $\left[\gamma^{-32} \mathrm{P}\right] \mathrm{ATP}$ and analyzed as above.

\section{In vitro binding assay}

Bacterially expressed and purified GST-fusion proteins (as above) were kept on beads and assayed for in vitro binding to Polo or hPlk1 by incubating with D-Mel or HeLa cell extracts in lysis buffer $(75 \mathrm{mM}$ K-HEPES at $\mathrm{pH} 7.5,150 \mathrm{mM} \mathrm{KCl}, 2 \mathrm{mM}$ $\mathrm{MgCl}_{2}, 2 \mathrm{mM}$ EGTA, 5\% glycerol, 0.1\% NP-40, 0.5\% Triton $\mathrm{X}-100$, Complete Protease Inhibitors [Roche]) for $30 \mathrm{~min}$ at $4{ }^{\circ} \mathrm{C}$, followed by five washes (5-10 min each) in lysis buffer. Beads were then heated in Laemmli SDS-PAGE sample buffer and analyzed by anti-Polo Western blot.

\section{Microscopy and flow cytometry}

Fixed cells stained by immunofluorescence were visualized on a Zeiss Axiovert 200 fluorescence microscope with Metamorph software. Movies of Polo-GFP D-Mel cells and GFP-Polo embryos were made on a Zeiss Axiovert 200 fluorescence microscope equipped with the PerkinElmer UltraVIEW confocal scanner and software. Fixed embryos were examined on a Nikon Optiphot fluorescence microscope equipped with a Bio-Rad MRC1024 confocal scanner and software. For flow cytometry, D-Mel cells were fixed in $70 \%$ ethanol, RNase-treated, and stained with propidium iodide. Results were analyzed with Summit (Dako Cytommation) and Multicycle (Phoenix Flow Systems).

\section{Acknowledgments}

We thank Michaela Scigelova and Gary Woffendin from ThermoFisher for performing the ETD mass spectrometry experiments for phosphorylation site mapping and Svenja Hester for protein identification. We thank Andrea Pereira for his generous gift of anti-Map205 antibodies, Hiro Ohkura for the map205null fly line, Matthew Savoian for precious help with the microcopy, and members of the Glover laboratory for useful advice and discussions. V.A. was supported by long-term fellowships from the European Molecular Biology Organization and from 
the Human Science Frontier Program. This work was also supported by CR-UK, BBSRC, and MRC grant G0501718.

\section{References}

Ahonen, L.J., Kallio, M.J., Daum, J.R., Bolton, M., Manke, I.A., Yaffe, M.B., Stukenberg, P.T., and Gorbsky, G.J. 2005. Pololike kinase 1 creates the tension-sensing 3F3/2 phosphoepitope and modulates the association of spindle-checkpoint proteins at kinetochores. Curr. Biol. 15: 1078-1089.

Alexandru, G., Uhlmann, F., Mechtler, K., Poupart, M.A., and Nasmyth, K. 2001. Phosphorylation of the cohesin subunit Scc1 by Polo/Cdc5 kinase regulates sister chromatid separation in yeast. Cell 105: 459-472.

Andersen, S.S. 2000. Spindle assembly and the art of regulating microtubule dynamics by MAPs and Stathmin/Op18. Trends Cell Biol. 10: 261-267.

Archambault, V., Zhao, X., White-Cooper, H., Carpenter, A.T., and Glover, D.M. 2007. Mutations in Drosophila Greatwall/ Scant reveal its roles in mitosis and meiosis and interdependence with polo kinase. PLoS Genet. 3: e200. doi: 10.1371/ journal.pgen.0030200.

Barr, F.A., Sillje, H.H., and Nigg, E.A. 2004. Polo-like kinases and the orchestration of cell division. Nat. Rev. Mol. Cell Biol. 5: 429-440.

Bettencourt-Dias, M., Giet, R., Sinka, R., Mazumdar, A., Lock, W.G., Balloux, F., Zafiropoulos, P.J., Yamaguchi, S., Winter, S., Carthew, R.W., et al. 2004. Genome-wide survey of protein kinases required for cell cycle progression. Nature 432: 980-987.

Bodenmiller, B., Mueller, L.N., Pedrioli, P.G.A., Pflieger, D., Juenger, M.A., Eng, J.K., Aebersold, R., and Tao, W.A. 2007. An integrated chemical, mass spectrometric and computational strategy for (quantitative) phosphoproteomics: Application to Drosophila melanogaster Kc167 cells. Mol. Biosyst. 3: 275-286.

Burkard, M.E., Randall, C.L., Larochelle, S., Zhang, C., Shokat, K.M., Fisher, R.P., and Jallepalli, P.V. 2007. Chemical genetics reveals the requirement for Polo-like kinase 1 activity in positioning RhoA and triggering cytokinesis in human cells. Proc. Natl. Acad. Sci. 104: 4383-4388.

Carmena, M., Riparbelli, M.G., Minestrini, G., Tavares, A.M., Adams, R., Callaini, G., and Glover, D.M. 1998. Drosophila polo kinase is required for cytokinesis. J. Cell Biol. 143: 659671.

Casenghi, M., Meraldi, P., Weinhart, U., Duncan, P.I., Korner, R., and Nigg, E.A. 2003. Polo-like kinase 1 regulates Nlp, a centrosome protein involved in microtubule nucleation. Dev. Cell 5: 113-125.

Cha, B., Cassimeris, L., and Gard, D.L. 1999. XMAP230 is required for normal spindle assembly in vivo and in vitro. $J$. Cell Sci. 112: 4337-4346.

Chen, F., Archambault, V., Kar, A., Lio, P., D'Avino, P.P., Sinka, R., Lilley, K., Laue, E.D., Deak, P., Capalbo, L., et al. 2007. Multiple protein phosphatases are required for mitosis in Drosophila. Curr. Biol. 17: 293-303.

Clarke, A.S., Tang, T.T., Ooi, D.L., and Orr-Weaver, T.L. 2005. POLO kinase regulates the Drosophila centromere cohesion protein MEI-S332. Dev. Cell 8: 53-64.

D'Avino, P.P., Savoian, M.S., Capalbo, L., and Glover, D.M. 2006. RacGAP50C is sufficient to signal cleavage furrow formation during cytokinesis. J. Cell Sci. 119: 4402-4408.

D'Avino, P.P., Archambault, V., Przewloka, M.R., Zhang, W., Lilley, K.S., Laue, E., and Glover, D.M. 2007. Recruitment of Polo kinase to the spindle midzone during cytokinesis re- quires the Feo/Klp3A complex. PLoS ONE 2: e572. doi: 10.1371/journal.pone.0000572.

Elia, A.E., Cantley, L.C., and Yaffe, M.B. 2003a. Proteomic screen finds $\mathrm{pSer} / \mathrm{pThr}$-binding domain localizing Plk1 to mitotic substrates. Science 299: 1228-1231.

Elia, A.E., Rellos, P., Haire, L.F., Chao, J.W., Ivins, F.J., Hoepker, K., Mohammad, D., Cantley, L.C., Smerdon, S.J., and Yaffe, M.B. 2003b. The molecular basis for phosphodependent substrate targeting and regulation of Plks by the Polo-box domain. Cell 115: 83-95.

Feng, Y., Hodge, D.R., Palmieri, G., Chase, D.L., Longo, D.L., and Ferris, D.K. 1999. Association of polo-like kinase with $\alpha-, \beta$ - and $\gamma$-tubulins in a stable complex. Biochem. J. 339: 435-442.

Fenton, B. and Glover, D.M. 1993. A conserved mitotic kinase active at late anaphase-telophase in syncytial Drosophila embryos. Nature 363: 637-640.

Ferris, D.K., Maloid, S.C., and Li, C.C. 1998. Ubiquitination and proteasome mediated degradation of polo-like kinase. Biochem. Biophys. Res. Commun. 252: 340-344.

Garcia-Alvarez, B., de Carcer, G., Ibanez, S., Bragado-Nilsson, E., and Montoya, G. 2007. Molecular and structural basis of polo-like kinase 1 substrate recognition: Implications in centrosomal localization. Proc. Natl. Acad. Sci. 104: 3107-3112.

Glover, D.M. 2005. Polo kinase and progression through M phase in Drosophila: A perspective from the spindle poles. Oncogene 24: 230-237.

Goldstein, L.S., Laymon, R.A., and McIntosh, J.R. 1986. A microtubule-associated protein in Drosophila melanogaster: Identification, characterization, and isolation of coding sequences. J. Cell Biol. 102: 2076-2087.

Golsteyn, R.M., Mundt, K.E., Fry, A.M., and Nigg, E.A. 1995. Cell cycle regulation of the activity and subcellular localization of Plk1, a human protein kinase implicated in mitotic spindle function. J. Cell Biol. 129: 1617-1628.

Goto, H., Kiyono, T., Tomono, Y., Kawajiri, A., Urano, T., Furukawa, K., Nigg, E.A., and Inagaki, M. 2006. Complex formation of Plk1 and INCENP required for metaphase-anaphase transition. Nat. Cell Biol. 8: 180-187.

Irminger-Finger, I., Laymon, R.A., and Goldstein, L.S. 1990. Analysis of the primary sequence and microtubule-binding region of the Drosophila 205K MAP. J. Cell Biol. 111: 25632572.

Kang, Y.H., Park, J.E., Yu, L.R., Soung, N.K., Yun, S.M., Bang, J.K., Seong, Y.S., Yu, H., Garfield, S., Veenstra, T.D., et al. 2006. Self-regulated Plk1 recruitment to kinetochores by the Plk1-PBIP1 interaction is critical for proper chromosome segregation. Mol. Cell 24: 409-422.

Kelm, O., Wind, M., Lehmann, W.D., and Nigg, E.A. 2002. Cell cycle-regulated phosphorylation of the Xenopus polo-like kinase Plx1. J. Biol. Chem. 277: 25247-25256.

Kumagai, A. and Dunphy, W.G. 1996. Purification and molecular cloning of Plx1, a Cdc25-regulatory kinase from Xenopus egg extracts. Science 273: 1377-1380.

Lane, H.A. and Nigg, E.A. 1996. Antibody microinjection reveals an essential role for human polo-like kinase 1 (Plk1) in the functional maturation of mitotic centrosomes. J. Cell Biol. 135: 1701-1713.

Lindon, C. and Pines, J. 2004. Ordered proteolysis in anaphase inactivates Plk1 to contribute to proper mitotic exit in human cells. J. Cell Biol. 164: 233-241.

Llamazares, S., Moreira, A., Tavares, A., Girdham, C., Spruce, B.A., Gonzalez, C., Karess, R.E., Glover, D.M., and Sunkel, C.E. 1991. polo encodes a protein kinase homolog required for mitosis in Drosophila. Genes \& Dev. 5: 2153-2165.

Logarinho, E. and Sunkel, C.E. 1998. The Drosophila POLO 
kinase localises to multiple compartments of the mitotic apparatus and is required for the phosphorylation of MPM2 reactive epitopes. J. Cell Sci. 111: 2897-2909.

Lowery, D.M., Mohammad, D.H., Elia, A.E., and Yaffe, M.B. 2004. The Polo-box domain: A molecular integrator of mitotic kinase cascades and Polo-like kinase function. Cell Cycle 3: 128-131.

Lowery, D.M., Clauser, K.R., Hjerrild, M., Lim, D., Alexander, J., Kishi, K., Ong, S.E., Gammeltoft, S., Carr, S.A., and Yaffe, M.B. 2007. Proteomic screen defines the Polo-box domain interactome and identifies Rock2 as a Plk1 substrate. EMBO J. 26: 2262-2273.

Macurek, L., Lindvist, A., Lim, D., Lampson, M.A., Klompmaker, R., Freire, R., Clouin, C., Taylor, S.S., Yaffe, M.B., and Medema, R.H. 2008. Polo-like kinase-1 is activated by aurora A to promote checkpoint recovery. Nature. doi: 10.1038 /nature 07185 .

Maiato, H., Sampaio, P., and Sunkel, C.E. 2004. Microtubuleassociated proteins and their essential roles during mitosis. Int. Rev. Cytol. 241: 53-153.

Moutinho-Santos, T., Sampaio, P., Amorim, I., Costa, M., and Sunkel, C.E. 1999. In vivo localisation of the mitotic POLO kinase shows a highly dynamic association with the mitotic apparatus during early embryogenesis in Drosophila. Biol. Cell. 91: 585-596.

Neef, R., Preisinger, C., Sutcliffe, J., Kopajtich, R., Nigg, E.A., Mayer, T.U., and Barr, F.A. 2003. Phosphorylation of mitotic kinesin-like protein 2 by polo-like kinase 1 is required for cytokinesis. J. Cell Biol. 162: 863-875.

Neef, R., Gruneberg, U., Kopajtich, R., Li, X., Nigg, E.A., Sillje, H., and Barr, F.A. 2007. Choice of Plk1 docking partners during mitosis and cytokinesis is controlled by the activation state of Cdk1. Nat. Cell Biol. 9: 436-444.

Nigg, E.A. 1993. Cellular substrates of p34(cdc2) and its companion cyclin-dependent kinases. Trends Cell Biol. 3: 296301.

Ohkura, H., Hagan, I.M., and Glover, D.M. 1995. The conserved Schizosaccharomyces pombe kinase plo1, required to form a bipolar spindle, the actin ring, and septum, can drive septum formation in G1 and G2 cells. Genes \& Dev. 9: 1059-1073.

Oshimori, N., Ohsugi, M., and Yamamoto, T. 2006. The Plk1 target Kizuna stabilizes mitotic centrosomes to ensure spindle bipolarity. Nat. Cell Biol. 8: 1095-1101.

Park, J.E., Park, C.J., Sakchaisri, K., Karpova, T., Asano, S., McNally, J., Sunwoo, Y., Leem, S.H., and Lee, K.S. 2004. Novel functional dissection of the localization-specific roles of budding yeast polo kinase Cdc5p. Mol. Cell. Biol. 24: 98739886.

Pearson, J., Godinho, S.A., Tavares, A., and Glover, D.M. 2006. Heterologous expression of mammalian Plk1 in Drosophila reveals divergence from Polo during late mitosis. Exp. Cell Res. 312: 770-781.

Pereira, A., Doshen, J., Tanaka, E., and Goldstein, L.S. 1992. Genetic analysis of a Drosophila microtubule-associated protein. J. Cell Biol. 116: 377-383.

Petronczki, M., Glotzer, M., Kraut, N., and Peters, J.M. 2007. Polo-like kinase 1 triggers the initiation of cytokinesis in human cells by promoting recruitment of the RhoGEF Ect2 to the central spindle. Dev. Cell 12: 713-725.

Qian, Y.W., Erikson, E., and Maller, J.L. 1999. Mitotic effects of a constitutively active mutant of the Xenopus polo-like kinase Plx1. Mol. Cell. Biol. 19: 8625-8632.

Rogers, S.L., Rogers, G.C., Sharp, D.J., and Vale, R.D. 2002. Drosophila EB1 is important for proper assembly, dynamics, and positioning of the mitotic spindle. J. Cell Biol. 158: 873-884.

Seki, A., Coppinger, J.A., Jang, C.Y., Yates, J.R., and Fang, G.
2008. Bora and the kinase aurora A cooperatively activate the kinase Plk1 and control mitotic entry. Science 320: 1655-1658.

Seong, Y.S., Kamijo, K., Lee, J.S., Fernandez, E., Kuriyama, R., Miki, T., and Lee, K.S. 2002. A spindle checkpoint arrest and a cytokinesis failure by the dominant-negative polo-box domain of Plk1 in U-2 OS cells. I. Biol. Chem. 277: 3228232293.

Sigrist, S.J. and Lehner, C.F. 1997. Drosophila fizzy-related down-regulates mitotic cyclins and is required for cell proliferation arrest and entry into endocycles. Cell 90: 671-681.

Sorokina, I. and Kashina, A. 2005. Archived gels as a tool for identification of protein complexes: Polo kinase cofractionates with Drosophila 205-kDa MAP and ncd in mitotic embryonic extracts. Anal. Biochem. 344: 155-157.

Sumara, I., Vorlaufer, E., Stukenberg, P.T., Kelm, O., Redemann, N., Nigg, E.A., and Peters, J.M. 2002. The dissociation of cohesin from chromosomes in prophase is regulated by Polo-like kinase. Mol. Cell 9: 515-525.

Sumara, I., Gimenez-Abian, J.F., Gerlich, D., Hirota, T., Kraft, C., de la Torre, C., Ellenberg, J., and Peters, J.M. 2004. Roles of polo-like kinase 1 in the assembly of functional mitotic spindles. Curr. Biol. 14: 1712-1722.

Sunkel, C.E. and Glover, D.M. 1988. polo, a mitotic mutant of Drosophila displaying abnormal spindle poles. J. Cell Sci. 89: 25-38.

Syka, J.E., Coon, J.J., Schroeder, M.J., Shabanowitz, J., and Hunt, D.F. 2004. Peptide and protein sequence analysis by electron transfer dissociation mass spectrometry. Proc. Natl. Acad. Sci. 101: 9528-9533.

van de Weerdt, B.C. and Medema, R.H. 2006. Polo-like kinases: A team in control of the division. Cell Cycle 5: 853-864.

van Vugt, M.A., van de Weerdt, B.C., Vader, G., Janssen, H., Calafat, J., Klompmaker, R., Wolthuis, R.M., and Medema, R.H. 2004. Polo-like kinase-1 is required for bipolar spindle formation but is dispensable for anaphase promoting complex/Cdc20 activation and initiation of cytokinesis. J. Biol. Chem. 279: 36841-36854.

Visintin, C., Tomson, B.N., Rahal, R., Paulson, J., Cohen, M., Taunton, J., Amon, A., and Visintin, R. 2008. APC/C-Cdh1mediated degradation of the Polo kinase Cdc5 promotes the return of Cdc14 into the nucleolus. Genes \& Dev. 22: 79-90.

Wong, O.K. and Fang, G. 2005. Plx1 is the 3F3/2 kinase responsible for targeting spindle checkpoint proteins to kinetochores. J. Cell Biol. 170: 709-719.

Wong, O.K. and Fang, G. 2007. Cdk1 phosphorylation of BubR1 controls spindle checkpoint arrest and Plk1-mediated formation of the 3F3/2 epitope. J. Cell Biol. 179: 611-617.

Xiang, Y., Takeo, S., Florens, L., Hughes, S.E., Huo, L.J., Gilliland, W.D., Swanson, S.K., Teeter, K., Schwartz, J.W., Washburn, M.P., et al. 2007. The inhibition of Polo kinase by Matrimony maintains G2 arrest in the meiotic cell cycle. PLOS Biol. 5: e323. doi: 10.1371/journal.pbio.0050323. 


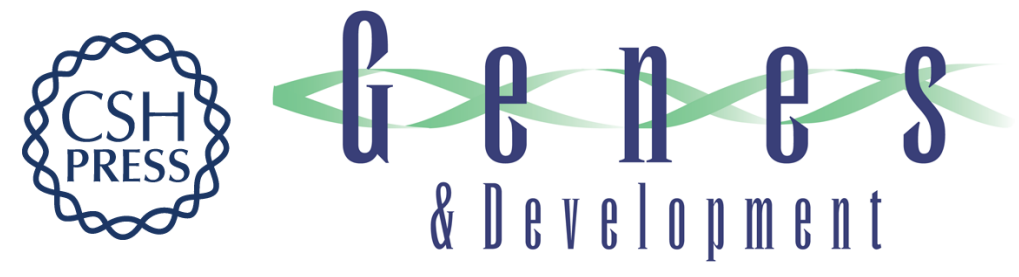

\section{Sequestration of Polo kinase to microtubules by phosphopriming-independent binding to Map205 is relieved by phosphorylation at a CDK site in mitosis}

Vincent Archambault, Pier Paolo D'Avino, Michael J. Deery, et al.

Genes Dev. 2008, 22:

Access the most recent version at doi:10.1101/gad.486808

Supplemental Material

References License

Email Alerting Service
http://genesdev.cshlp.org/content/suppl/2008/09/22/22.19.2707.DC1

This article cites 63 articles, 30 of which can be accessed free at: http://genesdev.cshlp.org/content/22/19/2707.full.html\#ref-list-1

Receive free email alerts when new articles cite this article - sign up in the box at the top right corner of the article or click here. 